\title{
Comparison of cotton swab to Whatman cellulose paper as media for examination of biomarkers from diabetic ulcers
}

\author{
Ronald W. Kartika ${ }^{1}$, Idrus Alwi ${ }^{2}$, Em Yunir ${ }^{2}$, Sarwono Waspadji ${ }^{2}$, Franciscus D. Suyatna ${ }^{3}$, \\ Suzzana Immanuel ${ }^{4}$, Saleha Sungkar ${ }^{5}$, Jusuf Rachmat ${ }^{6}$, Mirta Hediyati Reksodiputro, \\ Todung Silalahi ${ }^{8}$, Saptawati Bardosono ${ }^{9}$
}

\author{
${ }^{1}$ Doctoral Program in Medical Science Faculty of Medicine, Universitas Indonesia, \\ Jakarta Indonesia; Department of Surgery, Krida Wacana Christian University, \\ Jakarta, Indonesia \\ ${ }^{2}$ Department of Internal Medicine, Faculty of Medicine, Universitas Indonesia - \\ Cipto Mangunkusumo Hospital, Jakarta, Indonesia \\ ${ }^{3}$ Department of Clinical Pharmacology, Faculty of Medicine, Universitas Indonesia, \\ Jakarta, Indonesia \\ ${ }^{4}$ Department of Clinical Pathology, Faculty of Medicine, Universitas Indonesia - \\ Cipto Mangunkusumo Hospital, Jakarta, Indonesia \\ ${ }^{5}$ Department of Clinical Parasitology, Faculty of Medicine, Universitas Indonesia, \\ Jakarta, Indonesia \\ ${ }^{6}$ Department of Thoracic Cardiac and Vascular Surgery, Faculty of Medicine, \\ Universitas Indonesia, Jakarta, Indonesia \\ ${ }^{7}$ Departement Facial Plastic Reconstructive Division, Department \\ of Otorhinolaryngology, Faculty of Medicine, Universitas Indonesia, Cipto \\ Mangunkusumo Hospital, Jakarta, Indonesia; Department of Clinical Pharmacology, \\ Faculty of Medicine, Universitas Indonesia, Jakarta, Indonesia \\ ${ }^{8}$ Department of Internal Medicine, Krida Wacana Christian University, Jakarta, \\ Indonesia \\ ${ }^{9}$ Department of Nutrition, Faculty of Medicine, Universitas Indonesia, Jakarta, Indonesia
}

Submitted: 14 May 2021

Accepted: 20 June 2021

Arch Med Sci Civil Dis 2021; 6: e78-e83

DOI: https://doi.org/10.5114/amscd.2021.107850

Copyright @ 2021 Termedia \& Banach

\begin{abstract}
Introduction: Chronic wounds, including diabetic foot ulcers, require proper diagnostics. Proper wound care must be supported by proper diagnostic procedures, one of which is the diagnosis of biomarkers that accelerate and inhibit wound healing, consistent for wound analysis. Many researchers have stated the importance of gelatinase, collagenase and matrix metalloprotease (MMP) levels and their role in wound healing. A balance between vascular endothelial growth factor (VEGF), inflammatory cytokines, MMPs and tissue matrix metalloprotease inhibitors (TIMPs) is indispensable in the wound healing process. Previous test results showed Whatmann had the ability to carry out cytokines and proteases in diabetic foot ulcer (DFU). The purpose of this study was to compare the sampling technique with the cotton swab technique and use Whatman cellulose paper as a preliminary study. The biomarkers taken were VEGF, interleukin 6 (IL-6), MMP-9 and TIMP-1 as representatives of growth factors, markers of inflammation and protease enzymes. The aim of the study was to determine the standard for taking commonly used media practicality for the extraction of growth factor (GF) and protein from the wound surface.
\end{abstract}

Material and methods: Six DFU subjects were included in this study, and divided into two groups based on the method of topical extraction: the cotton group and the Whatman paper group. The swab method is to use a cotton swab on the center of the wound and rotate it about 3 times until the cotton

\section{Corresponding author:} Saptawati Bardosono Department of Nutrition Faculty of Medicine Universitas Indonesia Jakarta, Indonesia E-mail: tati.bardo@yahoo. com 
swab is wet. The Whatman method involves sticking Whatman paper to the wound surface, applying light pressure and leaving it for $15 \mathrm{~min}$. Cotton swabs and Whatman paper were placed in Eppendorf tubes with $1.5 \mathrm{~cm}^{3}$ of $\mathrm{NaCl}$ and processed by making lysates in an integrated laboratory. Furthermore, cotton swabs and Whatman paper containing wound fluid in the Eppendorf tube were centrifuged immediately at 10,000 rpm for $3 \mathrm{~min}$ at room temperature. The resulting cell-free supernatant was subjected to analysis by the ELISA method. VEGF, interleukin-6 and MMP-9 and TIMP-1 proteins were calculated by statistic analysis. Descriptive statistics were used to present the variable location and type of injury. Quantitative variables are summarized as mean with standard deviation (SD). The relationship between levels of biomarkers obtained from concurrently collected wound fluids (cotton swab and Whatman) was assessed using an independent $t$ test analysis using SPSS 2015 software, version 20.0

Results: There was a significant difference in material collection from cotton swabs compared to the material collection method with Whatman paper for MMP-9 protein $(p=0.028)$ and TIMP-1 $(p=0.030)$. Meanwhile, for VEGF and IL- 6 , there were no insignificant differences between the two methods of taking the material on the diabetic foot ulcers surface.

Conclusions: Diagnostic wound fluid in diabetic foot ulcers using a non-pressure wound swab (Levine technique) can be considered for the collection of wound fluid from diabetic foot ulcers for ELISA examination as a guideline for further treatment.

Key words: diabetic foot ulcer, Levine cotton swab, Whatman cellulose paper, ELISA biomarker.

\section{Introduction}

As one of the major chronic complications of diabetes, diabetic foot ulcers (DFU) are the leading cause of amputation and death. The clinical diagnosis and prognosis of DFU are objective until now inadequate. More comprehensive predictions of DFU risk and severity are needed to reflect new biomarkers for the effects of therapeutic interventions [1].

Because diabetes mellitus (DM) is associated with long-term microvascular and macrovascular complications, these complications need to be detected early. If DFU risk stratification can be obtained earlier in diabetic patients then morbidity and mortality may be reduced. Measurement characteristics and objective evaluation and biological markers are very important in early clinical diagnosis and prediction of DFU progression [2-4]. Recent advances in the identification of biomarkers that can be used as candidates for companion clinical examinations. Examination of inflammatory and angiogenesis biomarkers such as Vascular Endothelial Growth Factor (VEGF) that have been widely considered and used, also interleukins (ILS). Other factors that influence wound healing include protease enzymes such as matrix metalloprotease 9 (MMP-9) and tissue matrix metalloprotease-1 (TIMP-1) [5, 6].

In this review, a good biomarker collection medium is expected to assist in the accurate diagnosis of DFU. So far, various media have been used to obtain material for ELISA biomarker examinations. The purpose of this study was to compare the sampling technique with the cotton swab technique and use of Whatman cellulose paper as a preliminary study.

\section{Biomarkers of diabetic foot ulcers}

Sampling of wounds for analysis of biomarkers has become more prevalent in recent years with the advent of advanced molecular techniques. Although this type of sampling and analysis is primarily performed in research settings, health care companies foresee bringing such analytical techniques to the general health care practitioner as evidenced by the filing of numerous patents in this domain. Although the test methodologies themselves are important, the sample collected for use with these proposed tests is critical to obtain accurate and informative results. In most reported studies involving biomarker analysis, wound fluid is the sample analyzed [7-9]. Analysis of cytokines and matrix metalloproteinases in complex biological fluids requires a method that is sensitive, accurate and reliable by using classic solid-phase sandwich immunoassays, such as enzyme-linked immunosorbent assay (ELISA). Wound fluid diagnostics in diabetic foot ulcer is an important tool for simultaneous detection and quantification of multiple parameters in complex biological mixtures, such as plasma or wound fluid [10-12].

\section{Material and methods}

We conducted an open randomized controlled trial from January to July 2019. The study was approved by the Ethics Committee of the Faculty of Medicine of Universitas Indonesia ID 0855/UN2.F1/ ETIK/2018. Informed consent was obtained from the patients, including for the use of photographs. The study was conducted at Koja District Hospital. Patients with DFU and an average wound duration of 3 months, categorized as Wagner 2, ulcer $<40 \mathrm{~cm}^{2}$, were chosen as the subjects of the study.

Six DFU subjects were included in this study, and divided into two groups based on the meth- 
od of topical extraction: the cotton group and the Whatman paper group. To avoid bias, sampling was performed at the same time and adjacent wound locations in 6 DFU subjects with an area of $10-40 \mathrm{~cm}^{2}$. Location used by cotton swab to obtain a wound fluid sample, another location but close together using Whatman paper. The resulting cell-free supernatant was subjected to analysis by the ELISA method. VEGF, interleukin- 6 and MMP-9 and TIMP-1 proteins were calculated by statistical analysis using the independent $t$ test.

\section{Wound fluid collection methods}

Two different methods were used to collect wound fluid, namely with a cotton swab and with Whatman filter paper.

\section{Use of cotton swab to collect wound fluid}

The swab method is to use a cotton swab on the center of the wound and rotate it about 3 times until the cotton swab is wet. Each wound was swabbed using the Levine technique, involving rotation of a wound swab over a $1 \mathrm{~cm}^{2}$ area of the wound for $5 \mathrm{~s}$, using sufficient pressure to extract fluid from the inner part of the wound. The Levine swab method has several advantages over other methods, for example it is easier, and does not require a long time (Figure 1).

Cotton swabs were placed in Eppendorf tubes with $1.5 \mathrm{~cm}^{3}$ of $\mathrm{NaCl}$ and it was processed in an integrated laboratory. Cotton swabs and wound fluid were centrifuged immediately at 10,000 rpm for $3 \mathrm{~min}$ at room temperature. The precipitate of lysate was examined by the ELISA method [13].

\section{Use of Whatman cellulose paper to collect wound fluid}

The level of biomarkers measured in serum correlates well directly when compared with serum

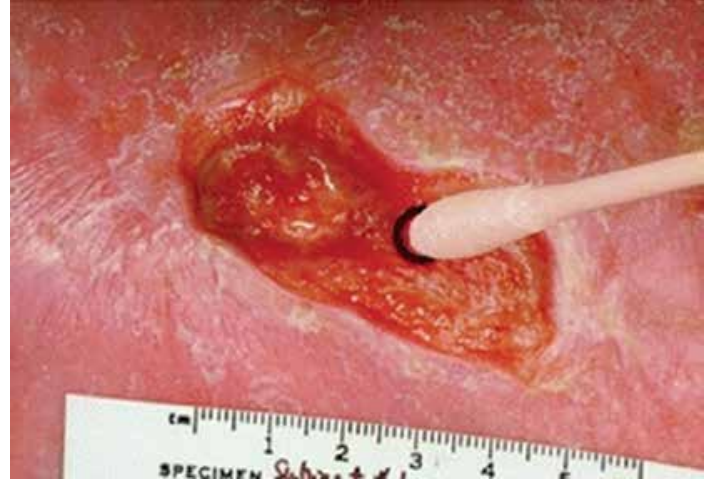

Figure 1. Collected wound fluid use cotton swab Levine technique

seen on whatman cellulose paper. Whatman 903 Protein Saver cards may catch more than $90 \%$ of all enzymes, using an automatic chemical analyzer (Figure 2).

Cellulose paper could potentially be used as a matrix for transporting and measuring alcohol biomarkers from field-based community studies. Previous studies from our group and other authors have also reported cellulose paper to be a reliable matrix for biochemical measurements.. After getting wet, the Whatman paper was placed in an Eppendorf tube with $1.5 \mathrm{~cm}^{3}$ of sodium chloride $0.9 \%$ as a buffer, followed by rotation at 10,000 rpm for $3 \mathrm{~min}$ at room temperature. After the supernatant was obtained, an ELISA examination was carried out $[14,15]$.

\section{Results}

The participants recruited for this study included 6 diabetes mellitus (DM) patients with 12 DFU Wagner 2 criteria. All the subjects met the inclusion criteria and were randomly allocated to two groups base on methods to collect the wound fluid by a cotton swab (group 1) and Whatman paper (group 2). The average age was 63.5 years, with a duration of

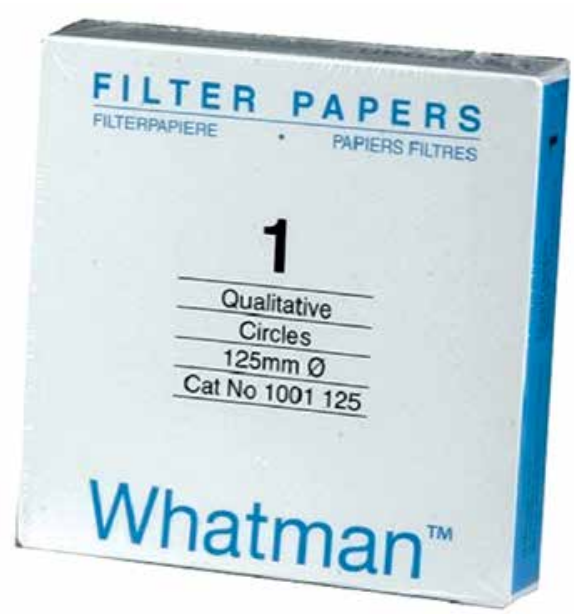

Figure 2. Whatman cellulose paper

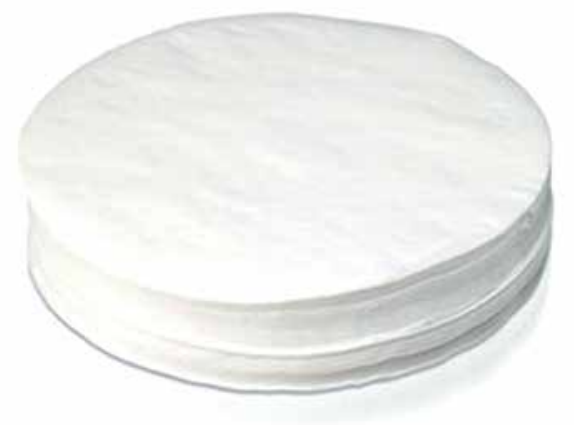


Table I. Wound characteristics based on intervention

\begin{tabular}{|lccc|}
\hline $\begin{array}{l}\text { Wound } \\
\text { characteristics }\end{array}$ & $\begin{array}{c}\text { Cotton } \\
\text { swab } \\
(n=6)\end{array}$ & $\begin{array}{c}\text { Whatman } \\
\text { paper } \\
(n=6)\end{array}$ & $\begin{array}{c}\text { Total } \\
n(\%)\end{array}$ \\
\hline \begin{tabular}{l} 
Wound location: \\
\hline Digiti
\end{tabular} & 3 & 3 & $6(50)$ \\
\hline \begin{tabular}{l} 
Forefoot \\
\hline Plantar
\end{tabular} & 1 & 1 & $2(16.6)$ \\
\hline Ankle & 1 & 1 & $2(16.6)$ \\
\hline Wound area [cm $\left.{ }^{2}\right]$ & 7.0 & 4.6 & $2(16.6)$ \\
\hline \begin{tabular}{l} 
Base of the wound: \\
\hline Dermis
\end{tabular} & 0 & 0 & $0.848^{\mathrm{a}}$ \\
\hline \begin{tabular}{l} 
Subdermis \\
\hline Fascia
\end{tabular} & 5 & 5 & $10(83.3)$ \\
\hline Muscle & 1 & 1 & $2(16.6)$ \\
\hline Secretion production: & & 0 & $0(0)$ \\
\hline \begin{tabular}{l} 
A little \\
\hline Moderate
\end{tabular} & 1 & 2 & $3(25)$ \\
\hline A lot & 4 & 4 & $8(66.7)$ \\
\hline Wound duration: & & 0 & $1(8.3)$ \\
\hline \begin{tabular}{l} 
Week-1 month \\
\hline$>1$ month
\end{tabular} & 4 & 4 & $8(66.6)$ \\
\hline
\end{tabular}

${ }^{a}$ Median (min.-max.), Mann-Whitney test.

DM of more than 5 years and a body mass index around $28.3 \mathrm{~kg} / \mathrm{m}^{2}$ (clinically obese). At the start of this study, the baseline data for all groups showed insignificant differences in blood glucose and $\mathrm{HbA}_{1 \mathrm{c}}$ levels as well as cholesterol levels.

Table I indicates that the location of most injuries was at the fingertips (43.3\%). All wounds were according to Wagner 2's criteria with subdermal wound bases (90\%), fascia (6.6\%) and dermis (3.3\%). Most of the secretions produced by DFU were moderate (63\%). Of the three treatment groups, the area of baseline DFU was $8.3 \pm 7.1 \mathrm{~cm}^{2}$ and there was no significant difference in the extent of DFU.

\section{Comparison of protein biomarker and enzyme entrapment in cotton swab and Whatman paper}

Table II shows the level of biomarkers VEGF and IL-6, from DFU wound fluid collected using a cotton swab compared with Whatman cellulose paper. There was not significant different protein wound fluid (VEGF, IL-6) and total protein entrapped in the cotton swab compared with Whatman cellulose paper.

However, Whatman cellulose paper had a significantly greater ability to withstand the enzymes MMP-9 $(p=0.028)$ and TIMP-1 $(p=0.030)$ compared with the cotton swab method (Table III).

\section{Discussion}

There are many ways to collect samples, including collecting wound fluid and performing an MMP-9 examination. There is also a skin biopsy on the DFU and checking for growth factors such as EGF, VEGF, TGF- $\beta$, FGF, EPQ2.

Howard et al. used a fine needle block biopsy and performed a paraffin block for TGF- $\beta 3$ examination. Another way is to use an adhesive patch skin biopsy and check for RNA, DNA, microbiome samples. New buccal swab brushes are carried out in children in Alzheimer's cases and DNA paternity checks are carried out. The method of examining buccal swab samples is cheaper, more practical, and easier.

Wound fluid is an interesting object of research because its composition can describe the state of the wound from the healing phase. The wound fluid consists of an inhomogeneous mixture consisting of various protein biomarkers and proteinase enzymes [16-18]. In the physiological course, the wound fluid should show changes in time (mainly related to the proteome). Several wound fluid enzymes such as proteases and their inhibitors, as well as growth factors and cytokines [19].

Ideally, wound fluid should be collected non-invasively. This is different from a tissue biopsy or technique defined as the gold standard in the evaluation of wound healing. There are several ways

Table II. Biomarker level of VEGF and IL-6 contained in cotton swab compared with Whatman paper

\begin{tabular}{|lccc|}
\hline Biomarkers & Cotton swab $(n=6)$ & Whatman paper $(n=6)$ & $P$-value \\
\hline VEGF & $382.4 \pm 176.7$ & $537.7 \pm 176.7$ & 0.142 \\
\hline IL-6 & $658.9 \pm 219.7$ & $518.1 \pm 259.4$ & 0.544 \\
\hline Protein total & $9.4 \pm 2.6$ & $7.0 \pm 2.4$ & 0.128 \\
\hline
\end{tabular}

Mean $\pm S D$, independent t-test

Table III. Enzyme of MMP-9 and TIMP-1 contained in cotton swab compared with Whatman paper

\begin{tabular}{|lccc|}
\hline Biomarkers & Cotton swab $(n=6)$ & Whatman paper $(n=6)$ & $P$-value \\
\hline MMP-9 & $8.6 \pm 0.6$ & $9.6 \pm 0.7$ & 0.028 \\
\hline TIMP-1 & $8.1 \pm 1.5$ & $10.2 \pm 1.4$ & 0.030 \\
\hline
\end{tabular}

Mean $\pm S D$, independent t-test. 
to collect the wound sample such as tissue biopsy, needle aspiration and quantitative swab $[16,19]$.

In this study, we found that there were no significant differences in growth factors and cytokines caught in cotton swabs compared to Whatman paper. This is due to the fact that cotton swabs have the ability to capture wound fluid quite adequately, even though the time of collection is quite short. This is because with the Levine technique with rotation of a cotton swab at the center of the wound, a fairly even protein sample will be obtained on the cotton swab.

However, in the study, cellulose Whatman paper showed the ability to bind to the proteinase enzyme (MMP-9) and its inhibitor (TIMP-1) directly compared to cotton [18].

Previous studies of biomarker samples were collected from Whatman filter paper. Some substances bind tighter to the filter paper and are eluted less efficiently than filter paper because of the cellulose content of Whatman filter paper [19].

Although the method using Whatman paper is more complete in capturing growth factors, cytokines and proteinases, sampling using Whatman filter paper is less practical. Besides taking a long time ( $\pm 1 \mathrm{~h})$, it also cannot be used on wound surfaces with holes $[20,21]$.

The gold standard for obtaining wound tissue or fluid is the biopsy technique because it has a fairly high accuracy. To overcome some side effects (such as pain, bleeding and infection) after an intervention biopsy procedure, researchers tried to use a cotton swab with the usual Levine technique to detect bacteria in DFU. There was no significant difference in the mean number of isolates per specimen between swabbing and deep tissue biopsy of diabetic foot ulcers of different grades [22, 23]. Ulcer swabbing, which is easier to perform and relatively noninvasive, could be a satisfactory clinical sampling technique compared with other techniques such as deep tissue biopsy [24-26].

In conclusion, cotton swab can be relied on to support the examination of biomarkers of growth factors, cytokines and protease enzymes in diabetic foot wounds classified as grade 2 . The use of a cotton swab for the collection of wound fluid examined by the ELISA method has several advantages over Whatman filter paper, including being more practical and requiring a shorter time, as well as being more economical.

\section{Acknowledgments}

This study was funded by Universitas Indonesia, Jakarta.

\section{Conflict of interest}

The authors declare no conflict of interest.

\section{References}

1. Alavi RG, Sibbald D, Mayer L, et al. Diabetic foot ulcers: part I. Pathophysiology and prevention. J Am Acad Dermatol 2014; 70: 19-20.

2. Aldrin G, Flores R, De Asis D. Microvascular complications and foot care: standards of medical care in diabetes-2020. Diabetes Care 2020; 43: 135-51.

3. Bao P, Kodra A, Tomic-Canic M, Golinko MS, Ehrlich HP, Brem $\mathrm{H}$. The role of vascular endothelial growth factor in wound healing. I Surg Res 2009; 153: 347-58.

4. Zubair M, Ahmad J. Role of growth factors and cytokines in diabetic foot ulcer healing: a detailed review. Rev Endocr Metab Disord 2019; 20: 207-17.

5. Avitabile S, Odorisio T, Madonna S, et al. Interleukin-22 promotes wound repair in diabetes by improving keratinocyte pro-healing functions. J Invest Dermatol 2014; 135: 2862-70.

6. Navarro-Gonzalez JF, Mora-Fernandez C. The role of inflammatory cytokines in diabetic nephropathy. J Am Soc Nephrol 2008; 19: 433-42.

7. Krisp C, Jacobsen F, McKay MJ, Molloy MP, Steinstraesser L, Wolters DA. Proteome analysis reveals antiangiogenic environments in chronic wounds of diabetes mellitus type 2 patients. Proteomics 2013; 13: 2670-81.

8. Jones JI, Nguyen TT, Peng Z, Chang M. Targeting MMP-9 in diabetic foot ulcers. Pharmaceuticals 2019; 12: 79-85.

9. Soo C, Shaw WW, Zhang X, Longaker MT, Howard EW, Ting K. Differential expression of matrix metalloproteinases and their tissue-derived inhibitors in cutaneous wound repair. Plast Reconstr Surg 2000; 105: 638-47.

10. Moses MA, Marikovsky M, Harper JW. Temporal study of the activity of matrix metalloproteinases and their endogenous inhibitors during wound healing. I Cell Biochem 1996; 60: 379-86.

11. Baker EA, Leaper DJ. Profile of matrix metalloproteinases and their tissue inhibitors in intraperitoneal drainage fluid: relationship to wound healing. Wound Rep Reg 2003; 11: 268-74.

12. Trengove NJ, Stacey MC, Macauley S, et al. Analysis of the acute and chronic wounds environments: the role of proteases and their inhibitors. Wound Rep Reg 1999; 7: 442-52.

13. Wysocki AB, Staiano-Coico L, Grinnell F. Wound fluid from chronic leg ulcers contain elevated levels of metalloproteinases MMP-2 and MMP-9. J Invest Dermatol 1993; 101: 64-8.

14. Yager DR, Zhang LY, Liang HX, Diegelmann RF, Cohen IK. Wound fluids from human pressure ulcers contain elevated matrix metalloproteinase levels and activity compared to surgical wound fluids. J Invest Dermatol 1996; 107: 743-8.

15. Yager DR, Stephen MC, Ward SI, Olutoye OO, Diegelmann RF, Cohen IK. Ability of chronic wound fluids to degrade peptide growth factors is associated with increased levels of elastase activity and diminished levels of proteinase inhibitors. Wound Rep Reg 1997; 5: 23-32.

16. Bullen EC, Longaker MT, Updike DL, et al. Tissue inhibitor of metalloproteinases- 1 is decreased and activated gelatinases are increased in chronic wounds. J Invest Dermatol 1995; 104: 236-40.

17. Vaalamo $M$, Weckroth $M$, Puolakkainen $P$, et al. Patterns of matrix metalloproteinase and TIMP-1 expression in chronic and normally healing human cutaneous wounds. Br J Dermatol 1996; 135: 52-9.

18. Ladwig GP, Robson MC, Liu R, Kuhn A, Muir DF, Schulz GS. Ratios of activated matrix metalloproteinase- 9 to tissue inhibitor of matrix metalloproteinase-1 in wound fluids 
are inversely correlated with healing of pressure ulcers. Wound Rep Reg 2002; 10: 26-37.

19. Trengove NJ, Bielefeldt-Ohmann H, Stacey MC. Mitogen ic activity and cytokine levels in non-healing and healing chronic leg ulcers. Wound Rep Reg 2000; 8: 13-25.

20. Poll EM, Kreitschmann-Andermahr I, Langejuergen Y, et al. Saliva collection method affects predictability of serum cortisol. Clin Chim Acta 2007; 3828: 15-9.

21. Rizwana $Q$, Vashney $M$, Singh A, et al. Use of filter paper to measure alcohol biomarkers among opioid-dependent patients on agonist maintenance treatment: a community-based study. Indian J Psychol Med 2019; 41: 529-34.

22. Rondas A, Schols J, Halfens G, Stobberingh E. Swab versus biopsy for the diagnosis of chronic infected wounds. Adv Skin Wound Care 2013; 26: 211-9.

23. Ramsay S, Cowan L, Davidson JM, Nanney L, Schults G. Wound samples:moving towards a standardsed method of collection and analysis. Int Wound J 2015; 13: 880-91.

24. Huovinen SM. Fine-needle aspiration biopsy, curettage, and swab samples in bacteriologic analysis of leg ulcers. Arch Dermatol 2012; 128: 856-7.

25. Johnson S, Lebahn F, Peterson LR, Gerding DN. Use of an anaerobiccollection and transport swab device to recover anaerobic bacteriafrom infected foot ulcers in diabetics. Clin Infect Dis 2014; 20: 289-90.

26. Little C. An overview of techniques used to measure wound area and volume. J Wound Care 2009; 18: 250-3. 